\title{
Prognostic factors for progression in atypical meningioma
}

\author{
Shakir I. Shakir, MD, ${ }^{1}$ Luis Souhami, MD, ${ }^{1}$ Kevin Petrecca, MD, PhD, ${ }^{2}$ Jose João Mansure, PhD, ${ }^{3}$ \\ Khushdeep Singh, MSc, ${ }^{4}$ Valerie Panet-Raymond, MD, ${ }^{1}$ George Shenouda, MD, PhD, ${ }^{1}$ \\ Amal A. Al-Odaini, MBBS, MSc, ${ }^{5}$ Bassam Abdulkarim, MD, PhD, ${ }^{1}$ and Marie-Christine Guiot, MD ${ }^{5}$
}

Departments of ${ }^{1}$ Radiation Oncology, ${ }^{2}$ Neurosurgery, ${ }^{3}$ Oncology Research Unit, ${ }^{4}$ Medical Physics, and ${ }^{5}$ Neuropathology, Cedars Cancer Centre, McGill University Health Centre, Montreal, Quebec, Canada

OBJECTIVE The optimal adjuvant management for atypical meningiomas remains controversial. The aim of this study was to review long-term outcomes to identify potential prognostic factors for disease progression.

METHODS From August 1992 to August 2013, 70 patients with atypical meningioma were treated at the authors' institution. Pathology revision was performed based on WHO 2007 criteria. Patients with multiple tumors, neurofibromatosis Type 2, or inadequate imaging follow-up were not eligible. The authors performed pre- and postoperative serial measurements of tumor volume from MRI. Age, sex, tumor location, bone involvement, brain invasion, mitotic figures, preoperative disease volume, extent of resection, tumor growth rates, use of adjuvant postoperative radiation therapy (PORT), and residual tumor volume at the time of radiation therapy (RT) were assessed by univariate and multivariate analysis to determine their potential impact on disease progression.

RESULTS Forty patients (57\%) underwent gross-total resection (GTR) and 30 (43\%) underwent subtotal resection (STR). PORT was delivered to 12 patients (30\%) with a GTR and in only 4 (13\%) with an STR. The 5-year progressionfree survival (PFS) rate for patients in the GTR group with or without PORT was $100 \%$ and $54.1 \%$, respectively $(p=$ 0.0058). PFS for patients in the STR group with or without PORT was $75 \%$ and $0 \%$, respectively $(p=0.0026)$. On multivariate analysis, STR and PORT were the only independent significant prognostic factors for disease progression with hazard ratios of $5.4873(95 \% \mathrm{Cl} 2.19-13.72, p=0.0003)$ and $0.0464(95 \% \mathrm{Cl} 0.0059-0.364, p=0.0035)$, respectively. Based on Youden's index statistic, a cutoff residual tumor volume of more than $8.76 \mathrm{~cm}^{3}$ at the time of RT was associated with worse PFS $(13.6 \%$ vs $56 \%, p=0.0079)$. Before receiving RT, the median relative and absolute growth rates and tumor doubling time for patients were $124.2 \% / y e a r, 4.8 \mathrm{~cm}^{3} / y e a r$, and 1.67 years, respectively. These indices changed after RT to $0.245 \% / y e a r,-0.09 \mathrm{~cm}^{3} / y e a r$, and -0.005 year, respectively $(p<0.05)$.

CONCLUSIONS In atypical meningioma, the use of PORT is associated with improved PFS even in patients who undergo GTR. Patients with residual tumor volume larger than $8.76 \mathrm{~cm}^{3}$ have an increased risk of disease progression and should be considered for early RT.

https://thejns.org/doi/abs/10.3171/2017.6.JNS17120

KEY WORDS atypical meningioma; Grade 2 meningioma; volumetric measurement; postoperative radiotherapy; prognostic factors; growth rate; oncology

$\mathrm{M}$ ENINGIOMAS, the most common primary CNS tumor in adults, are meningothelial cell neoplasms typically attached to the inner surface of the dura mater. ${ }^{3}$ These tumors include a wide spectrum of disease characteristics, ranging from benign lesions without local invasion (WHO Grade 1) to high-grade (WHO Grade 3, anaplastic) aggressive tumors characterized by a rapidly growing and destructive behavior.
Atypical, Grade 2 meningioma is a distinctive category of these tumors with a particular behavior in terms of growth pattern and local invasion. In their clinical course, approximately $40 \%$ tend to recur at 5 years if they are managed only with gross-total resection (GTR). ${ }^{1}$ The failure rate is higher and more frequent in the context of a subtotal resection (STR). ${ }^{1}$ The optimal postsurgical adjuvant management of such atypical lesions remains controversial.

ABBREVIATIONS $\mathrm{Cl}$ = confidence interval; $\mathrm{GTR}$ = gross-total resection; $\mathrm{HR}$ = hazard ratio; $\mathrm{PFS}$ = progression-free survival; $\mathrm{PORT}=$ postoperative $\mathrm{RT} ; \mathrm{RT}=$ radiation therapy; SRS = stereotactic radiosurgery; STR = subtotal resection.

SUBMITTED January 12, 2017. ACCEPTED June 19, 2017.

INCLUDE WHEN CITING Published online January 19, 2018; DOI: 10.3171/2017.6.JNS17120. 
For further characterization of potential variables with prognostic significance that might influence the outcome of this disease, we performed a retrospective review of patients with Grade 2 disease treated in our institution, looking at patient-, tumor-, and management-related parameters. By performing real volumetric tumor measurements, we also assessed absolute and relative tumor growth rates and tumor doubling time. In this paper, we report the results of this review.

\section{Methods \\ Patient Selection}

From August 1992 until August 2013, all patients with the diagnosis of WHO Grade 2 meningioma treated at our institution were reviewed. Patients were excluded from the study if they had 1 of the following criteria: history of neurofibromatosis Type 2, previous cranial radiation therapy (RT), multiple lesions, previously resected Grade 1 lesion that had transformed to a Grade 2 at time of recurrence, or inadequate patient or imaging follow-up (defined as a minimum 6-month follow-up, and at least 1 image showing progression or more than 2 images free of disease progression). Patients were included in the GTR group if there was no evidence of residual tumor on a follow-up MRI performed within 72 hours and they had a Simpson grade $^{21}$ between 1 and 3 . All other patients were considered to be in the STR group.

\section{Pathological Examination}

All pathological specimens of atypical meningioma from the cases treated at our institute between 1992 and 2013 were reviewed by 1 of the authors (M.C.G.). Except for a very small number of patients, the pathology of patients previously diagnosed with meningioma Grade 1 was not reviewed for potential inclusion in the study. The WHO 2007 pathological criteria were used to define Grade 2 disease. ${ }^{13}$ Tumors were classified as Grade 2 with the presence of brain invasion or a mitotic index of 4 or more per $10 \mathrm{hpf}$, either alone or combined. The diagnosis was also confirmed on the basis of the additive criteria of 3 or more of the 5 atypical histological features: area of geographic necrosis, nuclear pleomorphism, loss of architecture (sheeting), high cellularity, and the presence of foci of small hyperchromatic cells. Over 4 mitoses per 10 hpf was the most frequent diagnostic criteria (42 patients; Table 1). Brain invasion was noted in 25 cases, and 5 of them showed more than 4 mitoses per $10 \mathrm{hpf}$.

\section{Radiological Data}

All patients underwent pre- and postoperative MRI and during subsequent follow-up with intervals ranging from 3 to 6 months in the first 3 years, then every 10-12 months thereafter. Contrast-enhanced T1-weighted MR images were used to determine the tumor volume but were not necessarily performed on the same plane each time. The majority of MRI studies were performed using 3-5 $\mathrm{mm}$ slice thickness. We used standard radiological registry software (InteleViewer 4-9-1-P153) to measure tumor volume. Serial volume delineation of each tumor was performed by a single investigator (S.I.S.) using actual 3D volumetric sequences. Serial volumetric measurements were outlined on more than 900 image sequences.

We quantified changes in tumor size based on planimetric and volumetric measurements. Planimetric measurement was defined as a change in greatest linear diameter (one dimensional), any plane allowed. Measurements were performed by the primary investigator (S.I.S.). Planimetric failure was defined as the appearance of new enhancement at the surgical cavity for those patients with a GTR or an increase in any measurable tumor of more than $20 \%$ in any diameter, as per the definition of the Radiation Therapy Oncology Group protocol 0539. ${ }^{18}$ Volumetric failure was defined as a minimal change in volume of $20 \%$. This percentage was arbitrarily defined by us to correspond to the same percentage chosen for the definition of planimetric measurement progression.

\section{Treatment Modalities}

All patients underwent resection at our institution. GTR or STR was established by postoperative imaging and by the surgeon's assessment at the time of surgery based on the Simpson grading system. ${ }^{21}$ RT was delivered either in the adjuvant postoperative setting (postoperative RT, PORT) or at the time of tumor progression or growth (salvage). When used, RT was delivered to a gross target volume including any residual disease and the surgical cavity, based on a postoperative MR image or on MRI findings at the time of disease progression. Typically, a clinical target volume was created by adding a radial $1-\mathrm{cm}$ margin without cropping off the meningeal barriers and with inclusion of the inner plate of the skull, if applicable. An additional $3 \mathrm{~mm}$ was usually added for the planning target volume.

Adjuvant PORT was delivered to a median dose of 54 Gy (range 52.2-59.4 Gy) with daily fractionation at 1.8 Gy per fraction using 3D conformal techniques. Stereotactic radiosurgery (SRS) was not used as an adjuvant therapy. In case of tumor progression after RT, hypofractionated schedules or SRS were used, typically 40 Gy in 16 fractions or single-fraction SRS of 8 Gy or 12 Gy, respectively. One patient received concomitant temozolomide and RT after experiencing a third postsurgical failure.

\section{End Points and Statistical Analysis}

The primary end point of this review was to estimate the progression-free survival (PFS) after surgery. We analyzed several intrinsic (patient- and disease-related) and extrinsic (management-related) variables for possible prediction of progression, as follows: 1) age, 2) sex, 3) tumor location, 4) bone involvement, 5) preoperative tumor volume, 6) adjuvant PORT, 7) extension of the resection, 8) residual tumor volume at time of RT, 9) brain or pial invasion, and 10) number of mitotic figures per $10 \mathrm{hpf}$. Only variables with significance in the univariate analysis were included in the multivariate analysis.

The time lag of failure detection between planimetric and volumetric measurements was calculated based on the difference in the curve slopes between the 2 methods. A logistic regression model was used to evaluate the significance of this difference. 
TABLE 1. Baseline patient characteristics

\begin{tabular}{|c|c|}
\hline Parameter & No. of Patients (\%) \\
\hline \multicolumn{2}{|l|}{ Age (yrs) } \\
\hline$>65$ & $25(36)$ \\
\hline$\leq 65$ & $45(64)$ \\
\hline \multicolumn{2}{|l|}{ Sex } \\
\hline Male & $32(46)$ \\
\hline Female & $38(54)$ \\
\hline \multicolumn{2}{|l|}{ Location } \\
\hline Skull base & $19(27)$ \\
\hline Convexity & $10(14)$ \\
\hline Other locations & $41(59)$ \\
\hline \multicolumn{2}{|l|}{ Bone involvement } \\
\hline Present & $27(39)$ \\
\hline Absent & $43(61)$ \\
\hline \multicolumn{2}{|c|}{ Preop tumor volume $\left(\mathrm{cm}^{3}\right)$} \\
\hline$<30$ & $24(34)$ \\
\hline $30-60$ & $18(26)$ \\
\hline$>60$ & $28(40)$ \\
\hline \multicolumn{2}{|l|}{ Brain invasion } \\
\hline Absent & $45(64)$ \\
\hline Present & $25(36)$ \\
\hline \multicolumn{2}{|c|}{ Mitotic figures $>4 / \mathrm{hpf}$} \\
\hline$<4$ & $28(40)$ \\
\hline$\geq 4$ & $42(60)$ \\
\hline \multicolumn{2}{|l|}{ Resection } \\
\hline GTR & $40(57)$ \\
\hline STR & $30(43)$ \\
\hline \multicolumn{2}{|l|}{ PORT } \\
\hline Yes & $16(23)$ \\
\hline No & $54(77)$ \\
\hline
\end{tabular}

Absolute and relative tumor growth rates and tumor doubling time were measured for all patients using the calculations reported by Nakamura et al. ${ }^{14}$ with the following equations:

$$
\begin{aligned}
& \text { Absolute growth rate }\left(\mathrm{cm}^{3} / \text { year }\right)=\left(\mathrm{V}_{\text {latest }}-\mathrm{V}_{\text {initial }}\right) / \mathrm{t} \\
& \text { Relative growth rate }(\% / \text { year })=\left\{\left[\left(\mathrm{V}_{\text {latest }}-\mathrm{V}_{\text {initial }}\right)^{1 / t}\right]-1\right\} \\
& \quad \times 100
\end{aligned}
$$$$
\text { Doubling time }(\text { year })=\log _{2} /\left(\log \mathrm{V}_{\text {latest }}-\log \mathrm{V}_{\text {initial }}\right) \times \mathrm{t}
$$

where $\mathrm{V}_{\text {latest }}$ is the tumor volume measured on last followup, $V_{\text {initial }}$ is the tumor volume measured on the initial radiological study, and $t$ is the time interval between the initial and latest imaging studies (expressed in years). ${ }^{14}$ Comparison of growth parameters was performed before and after RT to estimate the potential effect of RT on the tumor growth kinetics using the variance ratio/F-test comparison method of the arithmetic means.

A descriptive analysis of the population was performed using the Stata (version 13, Stata Corp.) software package, for all statistical analyses. Using Youden's index ${ }^{19}$ statistic (sensitivity + specificity -1 ), the maximum value that represents optimal cutoff volume of residual disease and the correlation with disease control pattern was identified. PFS was calculated using Kaplan-Meier plots. Log-rank tests were used to evaluate differences in survival curves. The Cox proportional model was used during univariate and multivariate analyses to evaluate the prognostic significance of clinicopathological parameters. A p value < 0.05 was considered statistically significant. This review received approval from the institutional Ethics Board of McGill University Health Centre.

\section{Results}

\section{Demographic Data}

From August 1992 to August 2013, 93 patients were identified and their records reviewed. Twenty-three patients were excluded from the study for the following reasons: 4 patients because of insufficient follow-up, 4 were downgraded after pathology revision using the WHO 2007 criteria, 1 patient because of a transformation from WHO Grade 1 to Grade 2, 2 patients received previous cranial radiotherapy, 8 patients were diagnosed with neurofibromatosis Type 2 , and 4 patients had multiple lesions. Thus, 70 patients were available for final analysis. Table 1 shows the main patient characteristics. The median age of the group was 62 years (range 32-87 years). The median follow-up duration for all patients was 69 months (range 5.2-273.5 months). The median follow-up duration was 70.7 months for patients in the GTR group (range 7.7273.5 months) and 65.4 months (range 5.2-154.9 months) for patients in the STR group.

\section{GTR Versus STR, With or Without PORT}

Forty patients (57\%) underwent a GTR. Of these 40 patients, $12(30 \%)$ received adjuvant PORT. None of the patients receiving PORT developed a tumor progression, i.e., a 5-year PFS rate of $100 \%$ (Fig. 1A). Of the 28 patients (70\%) who did not receive PORT, 14 (50\%) experienced treatment failure at a median of 31 months (range 4-123 months), with a 5-year PFS rate of $54.1 \%(\mathrm{p}=0.0058)$. In the other 14 patients who underwent GTR without PORT and had their disease controlled, none had either bone involvement or skull-base tumor location.

Thirty patients $(43 \%)$ underwent STR and only 4 of them (13\%) received adjuvant PORT. Of these 4 patients, 3 (Fig. 1B) remained with disease controlled at a median follow-up duration of 72.13 months (range 58-108 months). The remaining patient receiving PORT after STR experienced treatment failure at 23 months posttherapy (both with bone involvement and skull-base disease location). Of the patients in the STR group who did not receive PORT, 1 patient, who had a near-total resection (without bone involvement or skull-base disease), has also remained recurrence-free at a follow-up time of 7 months at our institution (the patient was then followed elsewhere). All other 26 patients experienced treatment failure (median time for failure $=10$ months) and were managed with either subsequent surgeries or salvage RT later on. The 
A

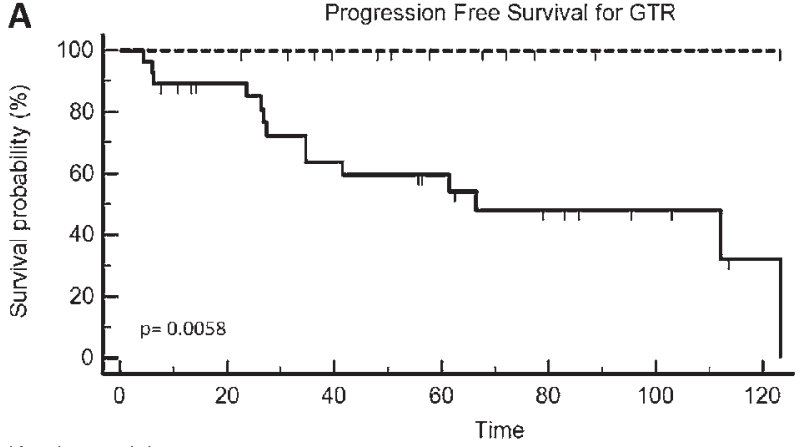

Number at risk

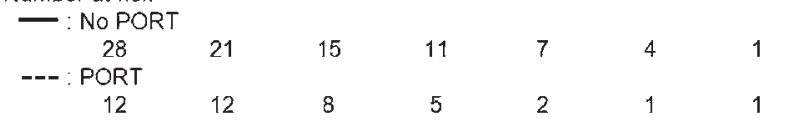

\section{B}

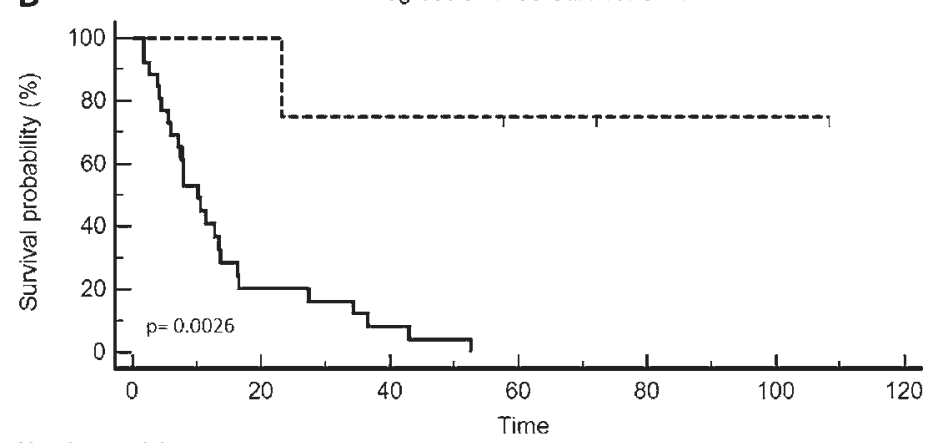

Number at risk

- No PORT

26
$--:$ PORT
4
5

4
3

\section{0}

2 $\begin{array}{lll}0 & 0 & 0 \\ 1 & 1 & 0\end{array}$

FIG. 1. PFS rates for patients who underwent GTR $(\mathbf{A})$ or STR $(\mathbf{B})$ with or without adjuvant PORT.

5-year PFS rate for patients in the STR group with or without PORT was $75 \%$ and $0 \%$, respectively $(\mathrm{p}=0.0026)$.

\section{Residual Volume}

For patients receiving RT at any time, the median residual volume at time of RT was $2.33 \mathrm{~cm}^{3}$ (range $0-83.5$ $\mathrm{cm}^{3}$ ). Using Youden's index statistic (Fig. 2), we observed that patients with residual volume of $>8.76 \mathrm{~cm}^{3}$ (seen in 11 patients) had a lower 5-year PFS rate of $13.6 \%$ in comparison with $56 \%$ observed in patients with a smaller volume $(\mathrm{p}=0.0079)$.

\section{Measurement of Failure}

We detected earlier tumor progression on follow-up imaging studies by performing volumetric rather than planimetric measurements. We observed a median time lag between the 2 detection methods of failure of 18 months (Fig. 3 ). At the time of diagnosis of disease progression, the median tumor volume was $4.89 \mathrm{~cm}^{3}$ on volumetric measurement compared with a median volume of $12.3 \mathrm{~cm}^{3}$ on planimetric measurement (i.e., the tumor volume will be already at least $50 \%$ larger by the time of planimetric detection; $p=0.0003$ ). Figure 4 shows examples of tumor changes based on planimetric versus volumetric measurements.

\section{Growth Pattern}

The relative growth rate, the absolute growth rate, and the tumor volume doubling time were determined to be $124 \%$ per year, $4.8 \mathrm{~cm}^{3}$ per year, and 1.67 years, respectively. All documented growth parameters were based on real volume contouring rather than estimation from planimetric values. For those patients receiving RT, there were changes in growth parameters, as follows: relative growth rate decreased to $0.25 \%$ per year ( $\mathrm{p}<0.001$ ), absolute growth rate dropped to $-0.09 \mathrm{~cm}^{3}$ per year $(\mathrm{p}=0.034)$ and, finally, the tumor size doubling time was slowed to -0.005 years $(p<0.001)$. The negative values represent inversion of growth, i.e., shrinkage (Table 2).

\section{Variables Associated With Tumor Progression}

On univariate analysis, male sex, skull base tumor loca- tion, bone involvement, preoperative tumor volume $>60$ $\mathrm{cm}^{3}$, brain invasion, STR, lack of PORT, and residual tumor volume larger than $8.76 \mathrm{~cm}^{3}$ significantly influenced disease progression. However, on multivariate analysis, only STR (negative) and PORT (positive) have significant impact on the progression rate, with hazard ratios (HRs) of $5.4873(95 \%$ CI $2.19-13.72, \mathrm{p}=0.0003)$ and $0.0464(95 \%$ CI $0.0059-0.364, \mathrm{p}=0.0035$ ), respectively (Table 3 ).

\section{Toxicity}

Generally, treatment was well tolerated. The Common Terminology Criteria for Adverse Effects grading scale (version 4) was used to capture and grade toxicity from RT. Three patients who presented with seizures prior to surgery and remained so after RT were not included in the RT-related toxicity group (improved subsequently with modification of anticonvulsive medication). There were no documented Grade 3 or 4 toxicities. Grade 1 or 2 toxicities were noted in 8 patients (radiotherapy attributed toxicity rate was 20\%) and included headache (4 patients), dizziness ( 3 patients) and paresthesia (1 patient). These toxicities were self-limited and managed with short-course corticotherapy. Two patients developed short-term memory impairment (1 of them had disease progression). Unfortunately, neurocognitive testing was not performed prior to surgery and therefore it was difficult to establish whether this toxicity was related to RT alone or a combination of factors. At the last follow-up evaluation there was no documentation of RT-induced brain necrosis. There is always the possibility that less detailed recording of toxicity has occurred, particularly for Grade 2 or 3 toxicities. However, the more serious (Grade 3 or 4) toxicities were more likely to have been accurately recorded. One patient experienced RT interruption and hospital admission due to an unrelated issue (acute renal failure).

\section{Discussion}

In patients undergoing a GTR for atypical meningiomas there remains a great deal of controversy and a persistent debate regarding optimal postoperative management. While some authors advocate the routine adjuvant use of 
A

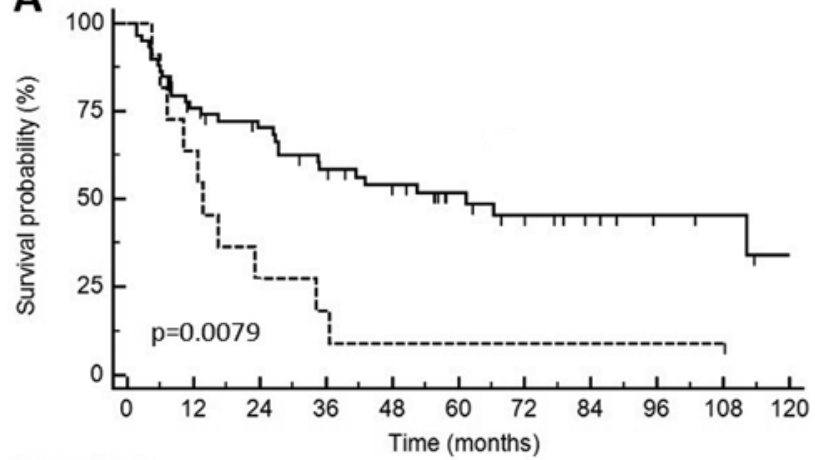

Number at risk

$-\leq 8.76 \mathrm{~cm}^{3}$

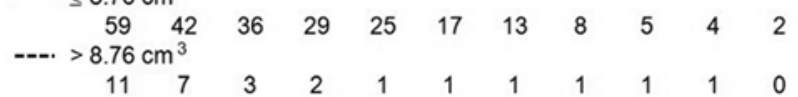

B Pre RT Volume

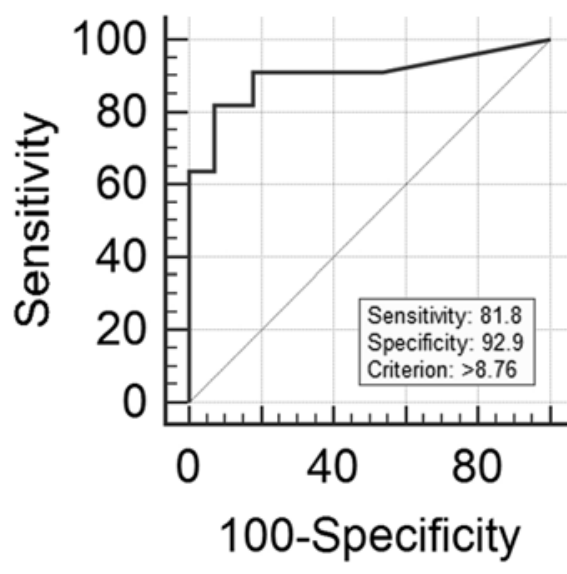

FIG. 2. A: PFS rates for all patients receiving RT based on the residual disease volume measured at the time treatment was delivered. B: Based on Youden's index statistic, a cutoff volume of $>8.76 \mathrm{~cm}^{3}$ was found to correlate with worse PFS.

PORT based on retrospective institutional reviews, ${ }^{1,2,11}$ others recommend close surveillance only.,22,23 A recent meta-analysis evaluating the benefit of RT in patients with atypical meningiomas after GTR suggests an important role for PORT. ${ }^{6}$ From 549 patients, Hasan et al. ${ }^{6}$ report that the progression rate doubled in patients not receiving PORT $(33.7 \%$ vs $15 \%, \mathrm{p}=0.005)$. However, there was no difference in the 5-year overall survival as most progressions were salvageable either by RT $(33 \%)$ or by surgery (67\%). Similar to other studies of a retrospective nature, this meta-analysis suffers from the usual flaws of such analyses including the lack of pathology review, inconsistent and small cohorts, treatment-selection bias, the varied definition of GTR and local failure, and short followup duration in some studies. While some authors report failure rates of $50 \%$ after GTR and observation, ${ }^{2}$ others ${ }^{23}$ report a failure rate of only $8.6 \%$, indicating the limitations of existing publications in defining the optimal role of PORT in this heterogeneous population.

Recently, the preliminary results of the Phase II Radiation Therapy Oncology Group protocol 0539 for patients with a new completely resected atypical meningioma or recurrent WHO Grade 1 meningioma irrespective of the extent of resection were reported in abstract form..$^{18}$ The study aim was to prospectively determine the 3 -year PFS rates after RT (54 Gy in 30 fractions) compared with an estimated predefined historical control of $70 \%$ with GTR alone. The 3-year PFS rate was 96\%, with 2 events observed: 1 patient with WHO Grade 2 and 1 patient with recurrent WHO Grade 1 died of disease or from undetermined cause, respectively. Treatments were well tolerated. The authors concluded that the results support the
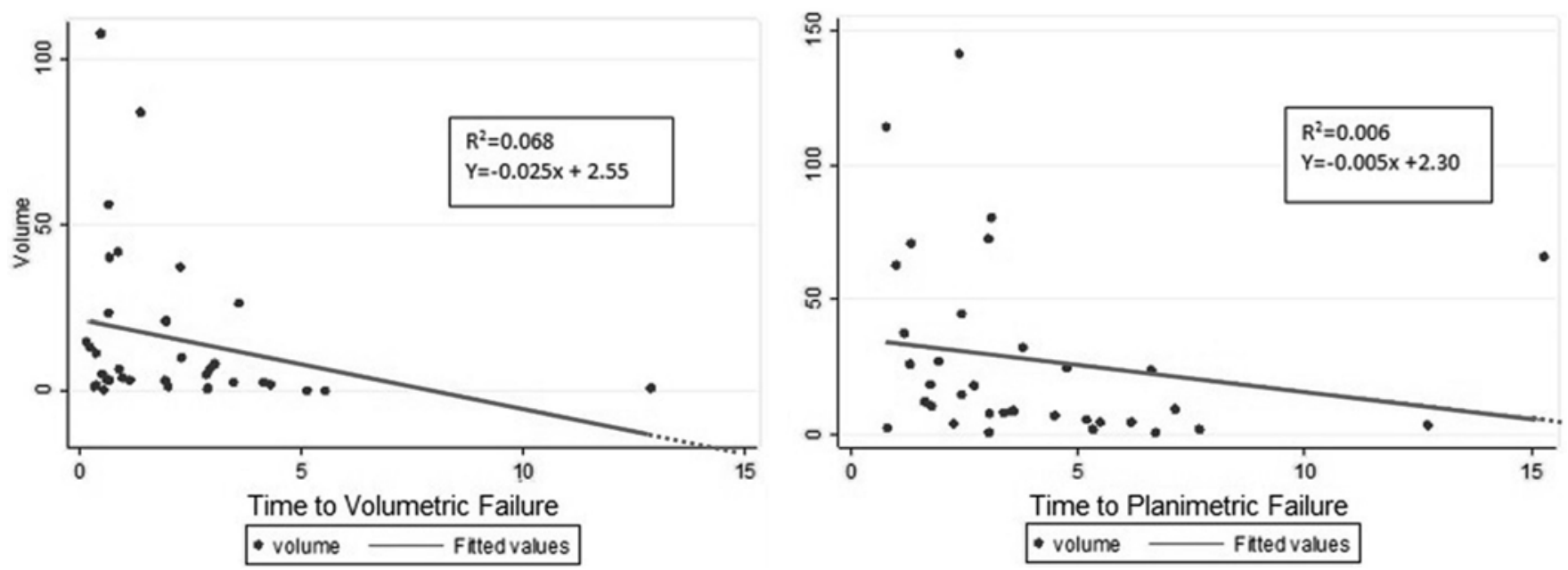

\section{Median time lag $(M \Delta t): 18$ months}

FIG. 3. Curves represent the volume of disease at the time of volumetric failure (left) and at the time of planimetric failure (right). The comparison of slopes shows a shorter detection time for failure using volumetric measurement, i.e., the bigger the slope the sooner it will cross the $\mathrm{x}$-axis and the earlier the detection will occur. 


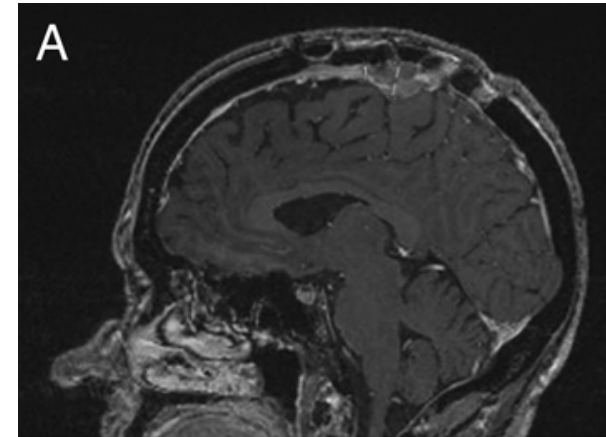

Base-line imaging:

Date: February 2003

Max-diameter: $1.45 \mathrm{~cm}$

Volume: $2.67 \mathrm{~cm}^{3}$

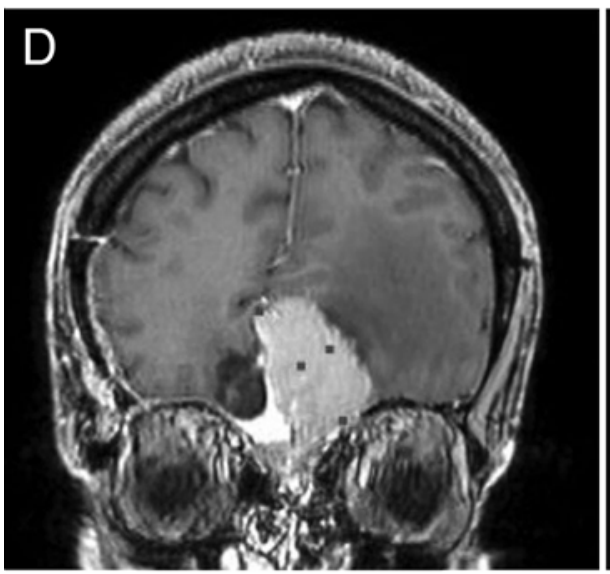

Base-line imaging:

Date: February 2010

Max-diameter: $3.84 \mathrm{~cm}$

Volume: $42.95 \mathrm{~cm}^{3}$

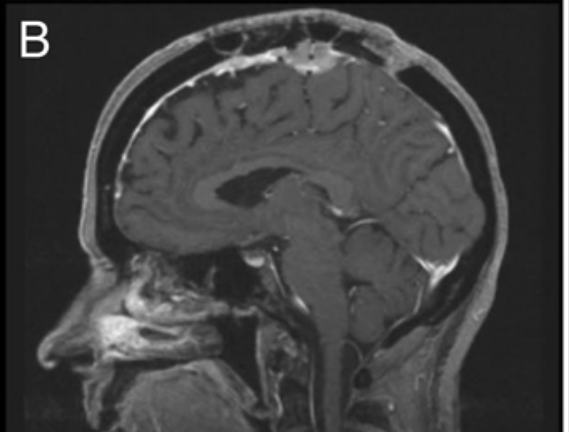

Volumetric failure

Date: September 2003

Max-diameter: $1.46 \mathrm{~cm}$

Volume: $3.92 \mathrm{~cm}^{3}$

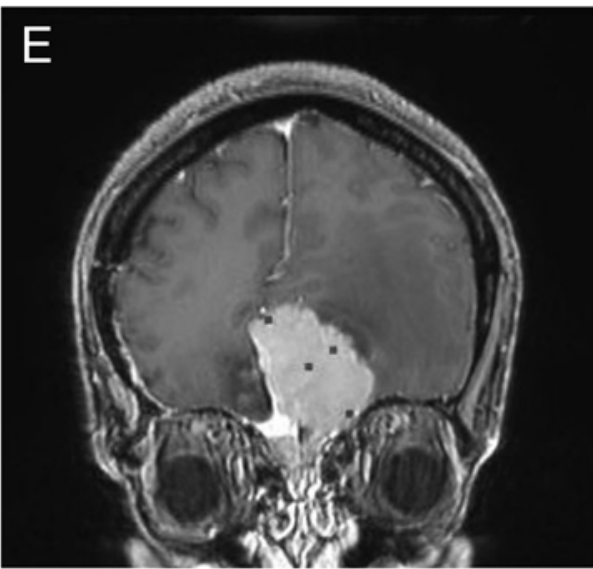

Volumetric failure

Date: June 2010

Max-diameter: $3.88 \mathrm{~cm}$

Volume: $55.98 \mathrm{~cm}^{3}$

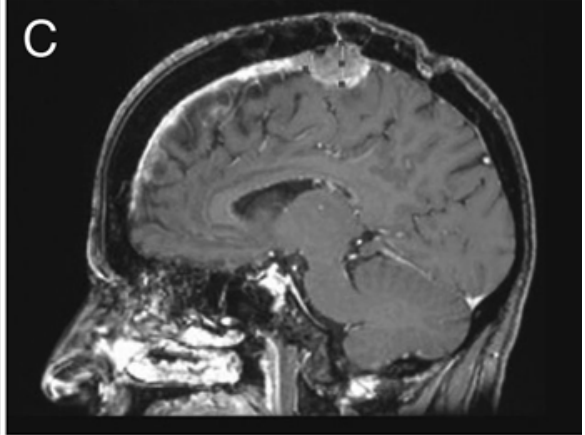

Planimetric failure

Date: April 2006

Max-diameter: $1.75 \mathrm{~cm}$

Volume: $8.76 \mathrm{~cm}^{3}$

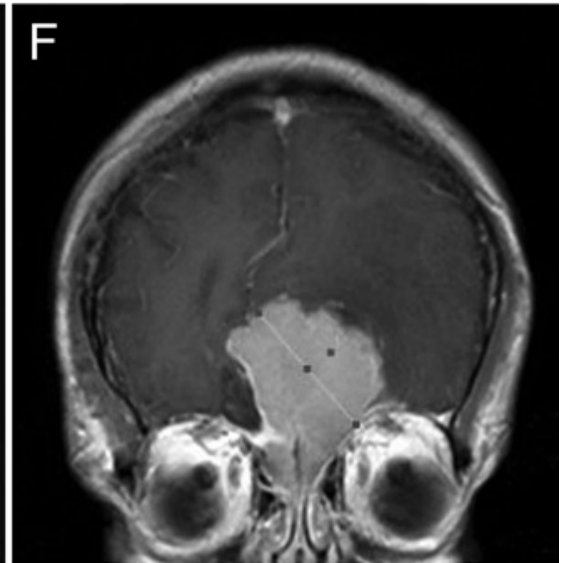

Planimetric failure

Date: February 2011

Max-diameter: $4.87 \mathrm{~cm}$

Volume: $70.88 \mathrm{~cm}^{3}$

FIG. 4. Changes in tumor size (from left to right) based on 3D or 2D measurements. Baseline image (A) shows a diameter of $1.45 \mathrm{~cm}$ and a volume of $2.67 \mathrm{~cm}^{3}$. In September 2003 (B) planimetric measurement showed a diameter of $1.46 \mathrm{~cm}$, whereas the volume was $3.92 \mathrm{~cm}^{3}$ (volumetric failure). Planimetric failure was diagnosed only in April 2006 (C) with a tumor diameter of 1.75 $\mathrm{cm}$, for a volume then of $8.76 \mathrm{~cm}^{3}$. Failure by planimetric measurement was detected 30 months after volumetric failure. A baseline image in February 2010 (D) shows a residual diameter of $3.84 \mathrm{~cm}$ and a volume of $42.95 \mathrm{~cm}^{3}$. In June 2010 (E), volumetric, but not planimetric, failure was determined (diameter $3.88 \mathrm{~cm}$, volume $55.98 \mathrm{~cm}^{3}$ ). Failure by 2D measurement was established 8 months later in February 2011 (F; diameter $4.87 \mathrm{~cm}$, volume $\left.70.88 \mathrm{~cm}^{3}\right)$.

use of PORT for GTR Grade 2 meningiomas. The European Organization for Research and Treatment of Cancer is currently conducting a Phase III trial of PORT versus observation in patients with atypical meningiomas after GTR $^{8}$ to ultimately define the role of PORT on tumor control post-GTR. Results of this randomized trial are eagerly

TABLE 2. Growth parameters for atypical meningioma before and after receiving $R T$

\begin{tabular}{lccc}
\hline \multicolumn{1}{c}{ Growth Parameter } & Before RT & After RT* & $\mathrm{p}$ Value \\
\hline Doubling time $(\mathrm{yrs})$ & 1.67 & -0.005 & $<0.001$ \\
\hline Relative growth rate $(\% / \mathrm{yr})$ & 124.2 & 0.245 & $<0.001$ \\
\hline Absolute growth rate $\left(\mathrm{cm}^{3} / \mathrm{yr}\right)$ & 4.8 & -0.09 & 0.034 \\
\hline
\end{tabular}

* Negative values represent tumor shrinkage rate or time.

$\dagger p$ value represents the significant difference in arithmetic means. awaited and until then this issue will remain unresolved. For those patients undergoing an STR, there appears to be a more general consensus on the value of PORT. ${ }^{4}$

In this retrospective review, the aim was to define potential variables associated with disease outcomes in patients receiving or not receiving PORT after resection of a Grade 2 meningioma. In univariate analysis from other series, several potential prognostic factors associated with disease progression have been identified in patients with atypical meningiomas. However, in most studies a limited sample size precluded a meaningful multivariate analysis. Reports of prognostic factors after multivariate modeling include a high mitotic index,,$^{10,12,23}$ Simpson surgical grading, ${ }^{5}$ sheeting, ${ }^{12}$ presence of necrosis ${ }_{12}^{12}$ pial and brain invasion, ${ }^{16,23}$ lack of PORT, ${ }^{12}$ and an STR ${ }^{12}$ Notwithstanding the fact that the number of patients studied in our review is relatively small preventing a definitive conclusion, a multivariate analysis showed that only the use of PORT and an 
TABLE 3. Univariate and multivariate analysis for all factors

\begin{tabular}{|c|c|c|c|c|c|c|}
\hline \multirow[b]{2}{*}{ Covariate } & \multicolumn{3}{|c|}{ Univariate Analysis } & \multicolumn{3}{|c|}{ Multivariate Analysis } \\
\hline & p Value & $\mathrm{HR}$ & $95 \% \mathrm{Cl}$ & p Value & $\mathrm{HR}$ & $95 \% \mathrm{Cl}$ \\
\hline \multicolumn{7}{|l|}{ Age group (yrs) } \\
\hline$\leq 65$ & Ref & & & & & \\
\hline$>65$ & 0.0552 & 1.8692 & $0.98-3.54$ & & & \\
\hline \multicolumn{7}{|l|}{ Sex } \\
\hline Female & Ref & & & & & \\
\hline Male & 0.0079 & 2.4302 & $1.26-4.67$ & 0.9917 & 1.0046 & $0.41-2.40$ \\
\hline \multicolumn{7}{|l|}{ Tumor location } \\
\hline Others & Ref & & & & & \\
\hline Convexity & 0.0708 & 0.2628 & $0.06-1.12$ & & & \\
\hline Skull base & 0.1200 & 1.6911 & $0.87-3.27$ & & & \\
\hline \multicolumn{7}{|l|}{ Bone involvement } \\
\hline No & Ref & & & & & \\
\hline Yes & 0.0231 & 2.0610 & $1.10-3.84$ & 0.1586 & 1.8379 & $0.78-4.28$ \\
\hline \multicolumn{7}{|c|}{ Preop volume $\left(\mathrm{cm}^{3}\right)$} \\
\hline$>60$ & Ref & & & & & \\
\hline$<30$ & 0.0469 & 0.4468 & $0.20-0.98$ & 0.7366 & 1.2007 & $0.41-3.48$ \\
\hline $30-60$ & 0.3411 & 0.6972 & $0.33-1.46$ & 0.5876 & 0.7749 & $0.30-1.94$ \\
\hline \multicolumn{7}{|l|}{ Brain invasion } \\
\hline No & Ref & & & & & \\
\hline Yes & 0.0130 & 2.2500 & $1.18-4.26$ & 0.4077 & 1.4608 & $0.59-3.58$ \\
\hline \multicolumn{7}{|c|}{ Mitotic figures per hpf } \\
\hline$\geq 4$ & Ref & & & & & \\
\hline$<4$ & 0.1236 & 1.6360 & $0.87-3.06$ & & & \\
\hline \multicolumn{7}{|l|}{ Surgery type } \\
\hline GTR & Ref & & & & & \\
\hline STR & $<0.0001$ & 5.9907 & $2.97-12.07$ & 0.0003 & 5.4873 & $2.19-13.72$ \\
\hline \multicolumn{7}{|l|}{ Adjuvant RT } \\
\hline No PORT & Ref & & & & & \\
\hline PORT & 0.0034 & 0.0514 & $0.007-0.37$ & 0.0035 & 0.0464 & $0.0059-0.364$ \\
\hline \multicolumn{7}{|c|}{ Pre RT volume $\left(\mathrm{cm}^{3}\right)$} \\
\hline$\leq 8.76$ & Ref & & & & & \\
\hline$>8.76$ & 0.0058 & 2.8163 & $1.34-5.88$ & 0.7546 & 0.8440 & $0.29-2.44$ \\
\hline
\end{tabular}

STR were associated with a significant impact on disease control.

Importantly, the volume of residual disease at the time of RT, whether given in the immediate postoperative period or after tumor recurrence, appears to bear a major impact on disease control. Patients with residual tumor volume greater than $8.76 \mathrm{~cm}^{3}$ had a significantly higher rate of failure, suggesting that RT should be offered earlier in the course of the disease for those with residual or progressive disease. This volume would roughly correspond to a sphere of $2.5 \mathrm{~cm}$ in diameter. The observation that volumes greater than $8.76 \mathrm{~cm}^{3}$ are associated with increased failure rate, even after the use of RT, leads to the question of how to optimally manage these patients. If feasible, re-excision should be considered prior to embarking on a course of RT.

Furthermore, our study shows that volumetric measurement is more accurate than, and superior to, 2D measure- ment and can detect disease progression much earlier with significantly smaller disease volume. Thus, we strongly recommend the volumetric method by delineating the residual disease volume and not by estimation from linear measurements to be used as the optimal approach when assessing imaging studies for disease recurrence or growth. Similar observations have been noted in other brain tumors. ${ }^{20,24}$

By using 3D modeling we were able to establish cell kinetic parameters before and after the use of RT. Previous studies on growth rates of meningiomas have been performed by other investigators. However, in most studies measurements were either performed in patients with asymptomatic or nonoperated lesions (radiological diagnosis) and most likely represent Grade 1 disease, ${ }^{14,15,17,25,26}$ or were completed postoperatively but with no grading reported. ${ }^{9}$ Nakamura et al. ${ }^{14}$ also determined absolute and relative growth rates and tumor volume doubling time in 
41 patients diagnosed with incidental meningiomas using equations similar to the ones performed in our study. Of interest, they established median absolute growth rate, relative growth rate, and tumor growth doubling time to be $0.796 \mathrm{~cm}^{3} /$ year, $14.6 \%$, and 21.6 years, respectively. These values are substantially lower than ours and likely represent the growth rates and doubling times of patients with Grade 1 disease.

Our study suffers the usual and expected limitations of retrospective reviews. There is inherent bias as treatment decision postsurgery was left to the discretion of the treating physician and not in a prospective, randomized manner. In addition, routine follow-up imaging studies were not prospectively established and most imaging studies were performed with 3-5 $\mathrm{mm}$ slice thickness, which may lead to uncertainty associated with tumor volume and planar computations. In addition, segmenting recurrent tumor volume from adjacent normally enhancing structures, such as venous sinuses, may pose further difficulty in accurately establishing disease progression. Despite these shortcomings, we believe that the information contained in this paper may serve as a guide to practicing professionals in the field of neurological sciences. Strengths of this study include the histopathological review of all patients by a neuropathologist using the 2007 WHO classification, the assessment of failure based on volumetric measurement, a relatively large sample size and, to our knowledge, the first time that growth rate parameters and tumor doubling time with or without RT has been determined in atypical meningiomas.

\section{Conclusions}

Our study provides new information on the importance of using volume measurement to determine tumor failure and also establishes parameters on tumor growth indices that may aid physicians in identifying patients who may benefit from more aggressive postoperative management, either in the adjuvant setting or at the time of recurrence. Although in our experience the use of PORT was associated with better disease control, its indication following a GTR for patients with atypical meningiomas remains unclear and further prospective, randomized studies are definitively needed to address this matter.

\section{References}

1. Aghi MK, Carter BS, Cosgrove GR, Ojemann RG, AminHanjani S, Martuza RL, et al: Long-term recurrence rates of atypical meningiomas after gross total resection with or without postoperative adjuvant radiation. Neurosurgery 64:56-60, 2009

2. Bagshaw HP, Burt LM, Jensen RL, Suneja G, Palmer CA, Couldwell WT, et al: Adjuvant radiotherapy for atypical meningiomas. J Neurosurg 126:1822-1828, 2017

3. Dolecek TA, Propp JM, Stroup NE, Kruchko C: CBTRUS statistical report: primary brain and central nervous system tumors diagnosed in the United States in 2005-2009. Neuro Oncol 14 (Suppl 5):v1-v49, 2012 (Erratum in Neuro Oncol 15:646-647, 2013)

4. Goldbrunner R, Minniti G, Preusser M, Jenkinson MD, Sallabanda K, Houdart E, et al: EANO guidelines for the diagnosis and treatment of meningiomas. Lancet Oncol 17:e383e391, 2016
5. Hammouche S, Clark S, Wong AH, Eldridge P, Farah JO: Long-term survival analysis of atypical meningiomas: survival rates, prognostic factors, operative and radiotherapy treatment. Acta Neurochir (Wien) 156:1475-1481, 2014

6. Hasan S, Young M, Albert T, Shah AH, Okoye C, Bregy A, et al: The role of adjuvant radiotherapy after gross total resection of atypical meningiomas. World Neurosurg 83:808815,2015

7. Jääskeläinen J: Seemingly complete removal of histologically benign intracranial meningioma: late recurrence rate and factors predicting recurrence in 657 patients. A multivariate analysis. Surg Neurol 26:461-469, 1986

8. Jenkinson MD, Javadpour M, Haylock BJ, Young B, Gillard H, Vinten J, et al: The ROAM/EORTC-1308 trial: Radiation versus Observation following surgical resection of Atypical Meningioma: study protocol for a randomised controlled trial. Trials 16:519, 2015

9. Jung HW, Yoo H, Paek SH, Choi KS: Long-term outcome and growth rate of subtotally resected petroclival meningiomas: experience with 38 cases. Neurosurgery 46:567-575, 2000

10. Klinger DR, Flores BC, Lewis JJ, Hatanpaa K, Choe K, Mickey B, et al: Atypical meningiomas: recurrence, reoperation, and radiotherapy. World Neurosurg 84:839-845, 2015

11. Komotar RJ, Iorgulescu JB, Raper DM, Holland EC, Beal $\mathrm{K}$, Bilsky MH, et al: The role of radiotherapy following gross-total resection of atypical meningiomas. J Neurosurg 117:679-686, 2012

12. Lee KD, DePowell JJ, Air EL, Dwivedi AK, Kendler A, McPherson CM: Atypical meningiomas: is postoperative radiotherapy indicated? Neurosurg Focus 35(6):E15, 2013

13. Louis DN, Perry A, Reifenberger G, von Deimling A, Figarella-Branger D, Cavenee WK, et al: The 2016 World Health Organization Classification of Tumors of the Central Nervous System: a summary. Acta Neuropathol 131:803-820, 2016

14. Nakamura M, Roser F, Michel J, Jacobs C, Samii M: The natural history of incidental meningiomas. Neurosurgery 53:62-71, 2003

15. Niiro M, Yatsushiro K, Nakamura K, Kawahara Y, Kuratsu J: Natural history of elderly patients with asymptomatic meningiomas. J Neurol Neurosurg Psychiatry 68:25-28, 2000

16. Nowak A, Dziedzic T, Krych P, Czernicki T, Kunert P, Marchel A: Benign versus atypical meningiomas: risk factors predicting recurrence. Neurol Neurochir Pol 49:1-10, 2015

17. Olivero WC, Lister JR, Elwood PW: The natural history and growth rate of asymptomatic meningiomas: a review of 60 patients. J Neurosurg 83:222-224, 1995

18. Rogers L, Zhang P, Vogelbaum MA, Perry A, Ashby L, Modi $\mathrm{J}$, et al: Intermediate-risk meningioma: initial outcomes from NRG Oncology/RTOG-0539. Int J Radiat Oncol Biol Phys 93 (3 Suppl):S139-S140, 2015

19. Ruopp MD, Perkins NJ, Whitcomb BW, Schisterman EF: Youden Index and optimal cut-point estimated from observations affected by a lower limit of detection. Biom J 50:419430, 2008

20. Sabit K, Panet-Raymond V, Souhami L, El Naqa I, Shenouda G, Zeitouni A, et al: Volumetric growth measurements of pituitary macroadenomas following surgical resection and indications for early adjuvant radiation therapy. Int J Radiat Oncol Biol Phys 90 (1 Suppl):S299-S300, 2014

21. Simpson D: The recurrence of intracranial meningiomas after surgical treatment. J Neurol Neurosurg Psychiatry 20:22-39, 1957

22. Stessin AM, Schwartz A, Judanin G, Pannullo SC, Boockvar JA, Schwartz TH, et al: Does adjuvant external-beam radiotherapy improve outcomes for nonbenign meningiomas? A Surveillance, Epidemiology, and End Results (SEER)-based analysis. J Neurosurg 117:669-675, 2012

23. Sun SQ, Kim AH, Cai C, Murphy RK, DeWees T, Sylvester 
P, et al: Management of atypical cranial meningiomas, part 1: predictors of recurrence and the role of adjuvant radiation after gross total resection. Neurosurgery 75:347-355, 2014

24. Vakilian S, Souhami L, Melançon D, Zeitouni A: Volumetric measurement of vestibular schwannoma tumour growth following partial resection: predictors for recurrence. J Neurol Surg B Skull Base 73:117-120, 2012

25. Yoneoka Y, Fujii Y, Tanaka R: Growth of incidental meningiomas. Acta Neurochir (Wien) 142:507-511, 2000

26. Zeidman LA, Ankenbrandt WJ, Du H, Paleologos N, Vick NA: Growth rate of non-operated meningiomas. J Neurol 255:891-895, 2008

\section{Disclosures}

The authors report no conflict of interest concerning the materi- als or methods used in this study or the findings specified in this paper.

\section{Author Contributions}

Conception and design: Souhami. Acquisition of data: Shakir, Guiot. Analysis and interpretation of data: Souhami, Shakir, Mansure, Singh, Guiot. Drafting the article: Souhami, Shakir. Critically revising the article: Souhami, Shakir, Petrecca, Mansure, Singh, Panet-Raymond, Shenouda, Abdulkarim, Guiot. Reviewed submitted version of manuscript: all authors. Approved the final version of the manuscript on behalf of all authors: Souhami. Statistical analysis: Mansure. Administrative/technical/material support: Petrecca, Al-Odaini, Guiot. Study supervision: Souhami.

\section{Correspondence}

Luis Souhami: McGill University Health Centre, Montreal, QC, Canada.luis.souhami@mcgill.ca. 